\title{
Photochromic Properties of 3-Deoxyanthocyanidin Pigments Extracted and Purified from Sorghum Grains
}

\author{
Mai Mrhara*, Kumiko TAsaki*, Yoshiumi Kohno** and Masashi Shibata*, ${ }^{*}$ \\ * School of Bioscience and Biotechnology, Tokyo University of Technology, 1404-1 Katakura-machi, Hachioji, Tokyo 192-0982, Japan \\ ** Department of Applied Chemistry and Biochemical Engineering, Faculty of Engineering, Shizuoka University, \\ 3-5-1 Johoku, Naka-ku, Hamamatsu, Shizuoka 432-8561, Japan \\ $†$ Corresponding Author, E-mail: mshibata@stf.teu.ac.jp
}

(Received December 1, 2017; Accepted February 8, 2018)

\begin{abstract}
With the aim of developing natural photochromic pigments, the selection of source materials and the extraction and purification methods for 3-deoxyanthocyanidins (3-DAs) were examined. Among the three raw materials derived from sorghum plants, the extract of Japanese Takakibi with 1, 3-butanediol contained the highest ratio of 3-DAs and exhibited the best photochromic properties. Furthermore, when fractions containing 3-DAs were collected from these materials using high-performance liquid chromatography (HPLC), the solutions decolored more readily in the light-shielding state and, hence, their photochromic performance improved drastically. Even Kaoliang food color, which had not previously shown photochromic behavior, showed photochromic performance after HPLC purification.
\end{abstract}

Key-words: Photochromic pigment, Sorghum, 3-deoxyanthocyanidins, Anthocyanin, HPLC

\section{Introduction}

Photochromic pigments are the ones that change their color when exposed to electromagnetic radiation. To date, many synthetic photochromic pigments have been studied. Some studies have also been conducted on naturally occurring photochromic pigments such as anthocyanidins and their derivatives, which have a lower environmental impact and lower human toxicity than synthetic pigments. Therefore, the industrial production of naturally occurring pigments is desirable. Luteolinidin, which is one of the 3-deoxyanthocyanidins (3-DAs) present in plants, has been reported to exhibit photochromic behavior in a watermethanol mixture ${ }^{1}$. 3-DAs are specific anthocyanins that have no hydroxyl group at the position 3 of the $\mathrm{C}$ ring (Fig. 1). We found that it was possible to repeatedly induce coloration and decoloration with luteolinidin in a nontoxic solution composed of weakly acidic water and 1,3-butanediol and that the other 3-DAs (e.g. apigeninidin, tricetininidin) also exhibit photochromic behavior ${ }^{2}$. However, the 3-DAs used in these studies were synthetic reagents and it was not clear from

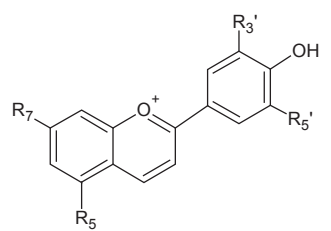

\begin{tabular}{|c|c|c|c|c|}
\hline Anthocyanidin & $\mathrm{R}_{3}{ }^{\prime}$ & $\mathrm{R}_{5}{ }^{\prime}$ & $\mathrm{R}_{5}$ & $\mathrm{R}_{7}$ \\
\hline Luteolinidin & $\mathrm{OH}$ & $\mathrm{H}$ & $\mathrm{OH}$ & $\mathrm{OH}$ \\
\hline Apigeninidin & $\mathrm{H}$ & $\mathrm{H}$ & $\mathrm{OH}$ & $\mathrm{OH}$ \\
\hline Tricetinidin & $\mathrm{OH}$ & $\mathrm{OH}$ & $\mathrm{OH}$ & $\mathrm{OH}$ \\
\hline
\end{tabular}

Fig. 1 Chemical structures of 3-deoxyanthocyanidins (3-DAs). prior studies whether natural 3-DAs actually extracted from plants exhibit the same photochromic properties.

Sorghum grains, which are used for food and livestock feed, are known to contain 3-DAs. Sorghum is primarily classified on the basis of the color of the seed coat. Among these types, black sorghum and brown sorghum are known to contain a large amount of 3-DAs ${ }^{3)}$. Besides 3-DAs, sorghums also contain anthocyanin pigments, which are not expected to show photochromic properties. In this study, we used raw materials derived from sorghums and investigated whether the pigments extracted from them actually exhibit photochromic properties.

\section{Experimental}

Three types of naturally derived samples (sorghum grains which are used for food or livestock feed) were used in this study. The first one is a commercial Kaoliang food color, which is extracted and purified from a seed of a type of sorghums (Sorghum nervosum Bess.). This powder was expected to contain a high quantity of anthocyanin pigments. The second is crushed black sorghum bran (Sorghum bicolor (L.) Moench, $\mathrm{T} x$ 430) from the United States, which is mainly used as cow feed and contains a large amount of 3-deoxyanthocyanidins (3-DAs). The third is the shell of the Japanese Takakibi (Sorghum bicolor Moench) seed from the Iwate Prefecture, which is a food grain with a shell that can easily and cheaply be obtained as waste material. The Japanese Takakibi was crushed with a food processor before 
use while the other samples were used, as provided, in their powder form.

1,3-butanediol (5 mL, special grade from Wako Pure Chemical Industries) was added to the sorghum grain powder samples $(0.1 \mathrm{~g})$ and the mixture was then ground by ball milling at $25^{\circ} \mathrm{C}$ for 1 day. The mixture was filtered (ca. $0.45 \mu \mathrm{m}$ pore size). The resultant 1,3-butanediol solution was mixed with weak acidic water $(\mathrm{pH} 4.1$, adjusted by a buffer consisting of phosphoric acid, boric acid, citric acid, and sodium hydroxide). A volume ratio of 1,3-butanediol/water $=$ 3 was used. The obtained pigment solutions were used after adjusting the concentration so that the maximum absorbance at the color peak (around $500 \mathrm{~nm}$ ) was 1.5.

UV-Vis spectra of the pigment solutions were measured under ultraviolet (UV) irradiation and light-shielding conditions. At first, the solutions were irradiated with UV light $\left(365 \mathrm{~nm}, 67 \mu \mathrm{W} / \mathrm{cm}^{2}\right)$ for $15 \mathrm{~min}$ (UV irradiation \#1). The solutions were then placed in the dark $\left(25^{\circ} \mathrm{C}\right)$ for 2 days (light shield storage). After that, the solutions were irradiated again with UV light for $15 \mathrm{~min}$ (UV irradiation \#2). The UVVis absorbance measurements of the solutions in each state were performed with a spectrophotometer (Jasco V650).

For analysis of the 3-DA content of sorghums, the pigment was dissolved in methanol and analyzed by highperformance liquid chromatography (HPLC, Agilent Technology system model 1260 Infinity). Methanol (HPLC grade, Fisher chemical) and acetic acid (super special grade, Wako Pure Chemical Industries, Ltd.) were used for analysis. Luteolinidin chloride and apigeninidin chloride standard samples for HPLC were obtained from Extrasynthese Co. The samples $(20 \mu \mathrm{L})$ were injected into the HPLC instrument using a $20-\mu \mathrm{L}$ loop. A reversed-phase C18 column $(4.5 \times 150 \mathrm{~mm}$, Zorbax ODS $)$ was used. Solvent A was water/acetic acid/methanol (81/9/10, vol. ratio) and solvent B was methanol/acetic acid (9/1). The elution program was a linear gradient from $0 \%$ to $100 \%$ of solvent B over $33 \mathrm{~min}$, followed by $100 \%$ solvent B for $5 \mathrm{~min}$, after which the column was cleaned by a linear gradient back to $0 \%$ of solvent $\mathrm{B}$ in $1 \mathrm{~min}$ and allowed to reequilibrate at $0 \%$ of solvent B for $15 \mathrm{~min}$. The flow rate was $1 \mathrm{~mL} / \mathrm{min}$ and detection was at $405 \mathrm{~nm}$.

Separation of the 3-DA components from sorghum samples by HPLC was performed as follows. Samples $(500 \mu \mathrm{L})$ were injected into the chromatograph using a $500-\mu \mathrm{L}$ loop. The reversed-phase $\mathrm{C}_{18}$ column $(9.4 \times 150 \mathrm{~mm}$, Zorbax ODS) was used. Other conditions were the same as those described for HPLC analysis. The HPLC-purified pigment solutions $(300 \mathrm{~mL})$ were added to 1,3-butanediol $(5 \mathrm{~mL})$, vacuum-dried in an evaporator $\left(65^{\circ} \mathrm{C}\right)$ to remove methanol and water. The photochromic performance of the purified pigment samples was examined in the same way as for samples without HPLC purification.

\section{Results and discussion}

The photochromic properties of 3-deoxyanthocyanidins (3-DAs) arise from a phototautomeric reaction that converts them from their trans-chalcone $(\mathrm{Ct})$ form to their cis-chalcone (Cc) form under UV irradiation. The reverse reaction, from the $\mathrm{Cc}$ to the $\mathrm{Ct}$ form, occurs in the absence of light as $\mathrm{Ct}$ is thermodynamically more stable. During UV irradiation, there is an increase in the colored species (the flavylium cation form, $\mathrm{AH}+$ ), because of equilibrium reactions involving the $\mathrm{Cc}, \mathrm{B}$, and $\mathrm{AH}+$ forms. Hence, the solution becomes red. When shielded from light, there is a decrease in the $\mathrm{AH}+$ form and decoloration occurs ${ }^{1}$.

Fig. 2 shows the absorption spectra of the Japanese Takakibi solution extracted with 1,3-butanedio. The red AH+ form, which is responsible for the photochromic properties, has an absorption peak at approximately $500 \mathrm{~nm}$. The colorless $\mathrm{Ct}$ form has an absorption peak at approximately $380 \mathrm{~nm}$. A preferable characteristic for a photochromic pigment is that the peak intensity of the colored type $\mathrm{AH}+$ form should become large during UV irradiation, whereas the peak should become smaller and preferably disappears during the time of light shielding. It is also necessary to repeat this change of intensity reversibly.

The ratios of the intensity of the $\mathrm{AH}+$ peak of the solutions under the light-shielded condition $\left(\mathrm{I}_{\text {dark }}\right)$ to that at the UV irradiated condition $\left(\mathrm{I}_{\mathrm{UV}}\right)$ are shown in Table 1. A lower $\mathrm{I}_{\text {dark }} / \mathrm{I}_{\mathrm{UV}}$ value corresponds to a higher photochromic performance. Among the three types of samples, the

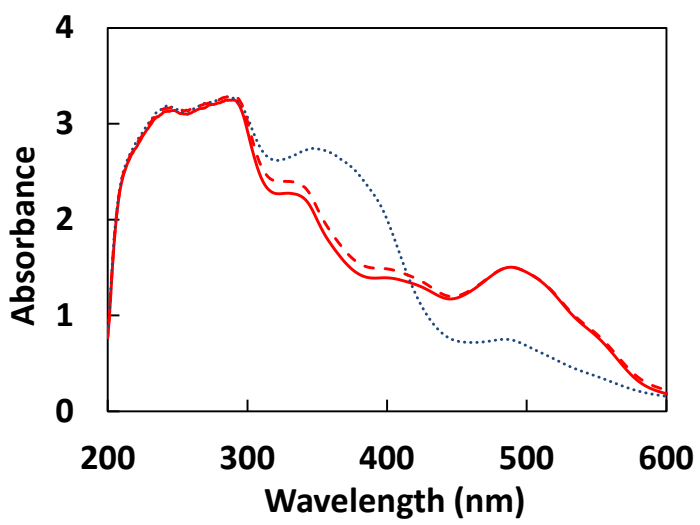

Fig. 2 UV-Vis spectra of the pigment solution extracted from Japanese Takakibi. Red solid line: UV irradiation \#1; Blue dotted line: light-shielded storage; Red dashed line: UV irradiation \#2.

Table 1 Ratio of the absorbance intensities of the $\mathrm{AH}+$ peak $\left(\mathrm{I}_{\mathrm{dark}} / \mathrm{I}_{\mathrm{UV}}\right)$.

\begin{tabular}{lcc}
\hline & Extracted & HPLC purified \\
\hline Japanese Takakibi & 0.50 & 0.31 \\
Black sorghum & 0.77 & 0.57 \\
Kaoliang food color & 0.94 & 0.71 \\
\hline
\end{tabular}


solutions of Japanese Takakibi and black sorghum showed photochromic properties (repeated coloring and decoloring). The ratio was 0.77 for black sorghum and 0.50 for Japanese Takakibi. Since the solution of Japanese Takakibi was more visibly decolorized, the pigment solution with Japanese Takakibi had better photochromic performance than that of black sorghum.

Compared with the other two samples, the Kaoliang food color showed little change in the absorbance between the shaded and UV-irradiated solutions. Additionally, there was almost no difference in the color of the solution found. This is presumed to be due to a larger presence of pigment molecules that do not cause discoloration in a light-shielding state (pigments other than 3-DAs) in the solution of Kaoliang food color, as compared to the Japanese Takakibi and Black Sorghum solutions.

The positions of the peaks attributed to the $\mathrm{AH}^{+}$forms of black sorghum, Kaoliang food color and Japanese Takakibi were 497, 484 and $488 \mathrm{~nm}$, respectively; the peak of black sorghum solution shifted to longer wavelengths compared to those of the other solutions.

Subsequently, in order to compare the composition of the 3-DAs in the extract solutions of the three raw materials, high-performance liquid chromatography (HPLC) analysis was performed. From chemical identification using standard reagents, luteolinidin $(\mathrm{Lt})$, which is red, and its $\mathrm{AH}+$ form peak at $512 \mathrm{~nm}$, were detected after $6.5 \mathrm{~min}$. Apigeninidin (Ap, orange, $505 \mathrm{~nm}$ ) was detected after $8.5 \mathrm{~min}$. Other 3-DAs were also detected in the vicinity of this range. Under this condition, photochromic pigments were detected from 6 to $10 \mathrm{~min}$. Common anthocyanidins (which do not have photochromic properties) were detected at longer retention times.

The total amount (ratio) of 3-DAs calculated from the detected peak area was 0.62 for Kaoliang food color, 0.79 for black sorghum, and 0.84 for Japanese Takakibi (Table 2). In accordance with the results of the photochromic test, Japanese Takakibi has the highest ratio of 3-DAs. As the ratio of 3-DAs in Kaoliang food color was the lowest, it was confirmed that its photochromic properties were inferior because there are many other coloring components (e.g., anthocyanins such as peonidin and malvidin) that do not decolorize, even when shielded from light.

Regarding the types of 3-DAs, the content of Ap is the largest in Japanese Takakibi (ratio is 0.78 ), while black sorghum mainly contained $\mathrm{Lt}$ and other 3-DA components (0.41, 0.29 respectively). For Kaoliang food color, Ap was abundant (0.52) (Table 2). The reason for the AH+ peak of black sorghum being located at a longer wavelength compared to that of the other samples is believed to be that black sorghum mainly contains luteolinidin, in contrast to the other samples that contained mainly Apigeninidin.

Even in Japanese Takakibi solution, which had the best photochromic performance among the three samples, the red color of the solution is rather intense in the light shielding state, as compared with the solutions composed of synthetic 3-DA reagents ${ }^{2}$. Hence, it is necessary to further improve the performance of the naturally occurring pigments for practical usage. If the 3-DA components can be isolated and extracted from the solution, the molecules that cannot be decolorized are removed and the photochromic properties are expected to improve. Furthermore, depending on the composition of the 3-DAs, it is expected that the color and photochromic performance of the solution will be different depending on the type of plant sources.

In order to isolate and obtain only 3-DAs, only the fractions in the range 6-10 min were collected. After HPLC purification, the $\mathrm{I}_{\mathrm{dark}} / \mathrm{I}_{\mathrm{UV}}$ ratio of all samples decreased, indicating that the photochromic performance of the products from all three sorghums improved. Compared to the solution before HPLC purification, the color erasure of these fractionated solutions became more conspicuous in the lightshielding state. Even the Kaoliang food color, which did not previously show clear photochromic performance, was shown to be a good photochromic solution after the HPLC fractionation treatment (Fig. 3, Table 1). Japanese Takakibi's photochromic performance was the best after simple
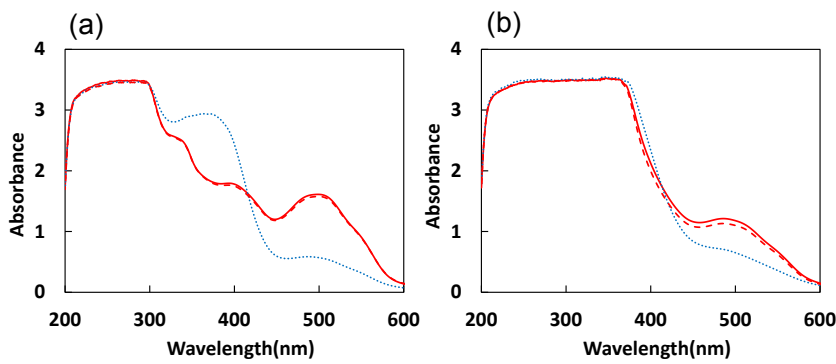

Fig. 3 UV-Vis spectra of (a) Japanese Takakibi solution and (b) Kaoliang food color solution purified by HPLC. Red solid line: UV irradiation \#1; Blue dotted line: lightshielded storage; Red dashed line: UV irradiation \#2.

Table 2 Quantity of 3-deoxyanthocyanidins extracted from sorghums.

\begin{tabular}{lcccc}
\hline & Luteolinidin & Apigeninidin & Other 3-DAs & Total 3-DAs \\
\hline Japanese Takakibi & 0.00 & 0.78 & 0.06 & 0.84 \\
Black sorghum & 0.41 & 0.09 & 0.29 & 0.79 \\
Kaoliang food color & 0.03 & 0.52 & 0.07 & 0.62 \\
\hline
\end{tabular}

The ratio was estimated from the peak areas obtained by HPLC analysis. 
extraction and improved further after HPLC.

By separating and purifying the natural products by HPLC, it was possible to obtain a practically usable photochromic pigment.

\section{Conclusion}

A photochromic pigment solution was obtained from a natural product (sorghum). The extract of Japanese Takakibi with 1,3-butanediol, which contained a high ratio of 3-deoxyanthocyanidins (3-DAs), exhibited the best photochromic properties. By separation and purification of only 3-DAs from sorghums using HPLC, the solutions decolored more readily in the light-shielding state and, hence, the photochromic performance of the three samples improved drastically. This indicates that industrially usable photochromic pigments were successfully obtained from a natural plant.

\section{References}

1) M. J. Melo, S. Moura, A. Roque, M. Maestri, F. Pina: J. Photochem. Photobio. A, 135 (33), (2000).

2) Y. Yagishita, M. Mihara, Y. Kohno, M. Shibata: J. Food Sci., 81 (2950), (2016).

3) J. M. Awika, L. W. Rooney, R. D. Waniska: J. Agric. Food Chem., 52 (4388), (2004). 